\title{
Protocolos de rehabilitación cardiaca mediante realidad virtual: revisión sistemática
}

\author{
Virtual reality protocols in cardiac rehabilitation: systematic review
}

\author{
Borja Alsina ${ }^{1}$ y José M. Cancela ${ }^{1,2 *}$
}

${ }^{1}$ Facultad de Ciencias de la Educación y del Deporte, Universidad de Vigo; ${ }^{2}$ Grupo de Investigación HealthyFit, Instituto de Investigación Sanitaria Galicia Sur, Servicio Gallego de Salud. Vigo, España

\section{Resumen}

Introducción: El desarrollo de protocolos de rehabilitación cardiaca tradicional proporciona grandes beneficios para la salud a pesar de las limitaciones espaciales y funcionales que generan. Los sistemas de realidad virtual han sido objeto de gran interés en la rehabilitación cardiaca debido a los beneficios que aportan, a la motivación de los pacientes y a la reducción de los plazos. Objetivo: Evaluar la eficacia de los sistemas de realidad virtual en la aplicación de programas de rehabilitación cardiaca. Método: Se realizó una revisión sistemática sobre el uso de los sistemas de realidad virtual en rehabilitación cardiaca y su efecto. En la búsqueda se incluyeron las bases de datos Scopus, Sport-Discus, PubMed-Medline, Web of Science y Dialnet. Resultados: De 280 artículos identificados, ocho fueron escogidos de acuerdo con los criterios de inclusión. Un total de 872 pacientes fueron analizados. Los resultados mostraron diferencias significativas a favor de las terapias con realidad virtual, en la adherencia y en mejores niveles de los indicadores de capacidad y de potencia aeróbica. Conclusiones: Se confirma que la aplicación de sistemas de realidad virtual en programas de rehabilitación cardiaca favorece la adherencia al programa y genera mejoras en los indicadores físicos.

Palabras clave: Realidad virtual. Rehabilitación cardiaca. Protocolos.

\begin{abstract}
Introduction: The development of traditional cardiac rehabilitation protocols provides great health benefits despite the spatial and functional limitations that they generate. Virtual reality systems have been the object of great interest in cardiac rehabilitation due to the benefits they provide, the motivation of patients and the reduction of time limits. Objective: To evaluate the effectiveness of virtual reality systems in the application of cardiac rehabilitation programs. Method: A systematic review was carried out to evaluate the use of virtual reality systems in cardiac rehabilitation programs. The search included databases such as Scopus, Sport-Discus, PubMed-Medline, Web of Science, and Dialnet. Results: Of 280 identified articles, eight were chosen based on the inclusion criteria. A total of 872 patients were analyzed. The results changed differences in favor of virtual reality therapies, in the motivation to develop the program, reduction in hospitalization time, better levels of capacity and aerobic power markers. Conclusions: We can confirm the evidence of the application of virtual reality technologies in cardiac rehabilitation programs causing improvements in aerobic capacity, vital capacity or in the level of effort that patients with cardiac pathology endure.
\end{abstract}

Key words: Virtual reality. Cardiac rehabilitation. Protocols.

\section{Correspondencia:}

*José M. Cancela

E-mail: chemacc@uvigo.es
Disponible en internet: 07-01-2022

Rev Colomb Cardiol. 2021;28(6):616-629

www.rccardiologia.com 0120-5633 / @ 2021 Sociedad Colombiana de Cardiología y Cirugía Cardiovascular. Publicado por Permanyer. Este es un artículo open access bajo la licencia CC BY-NC-ND (http://creativecommons.org/licenses/by-nc-nd/4.0/). 


\section{Introducción}

Puede resultar complicado establecer unas cifras exactas sobre los distintos orígenes de las enfermedades cardiacas debido a la escasez de estudios epidemiológicos y la gran variabilidad de factores existentes (edad, raza, sexo, etc.), pero sin necesidad de especificar en ello, las enfermedades coronarias son una de las principales causas de mortalidad en nuestro actual mundo industrializado ${ }^{1}$. La intervención ante estas enfermedades coronarias se efectúa desde un punto de vista multidimensional fundamentada en una supervisión hospitalaria. La aplicación de programas de rehabilitación cardiaca proporciona grandes beneficios para la salud de estos pacientes, ya que reduce la mortalidad $^{2}$. Estos programas están diseñados y supervisados por distintos profesionales, como cardiólogos, psiquiatras, psicólogos, médicos rehabilitadores, fisioterapeutas y enfermeras, y su aplicación ha demostrado mejorar el pronóstico de la enfermedad y la calidad de vida del paciente ${ }^{1}$. Los programas de ejercicio físico orientados a esta población se fundamentan en un componente aeróbico cuya duración, frecuencia e intensidad varían en función de los análisis previos o de la enfermedad intervenida.

Las muertes por enfermedad coronaria se han visto reducidas gracias a la aplicación de tratamientos basados en el desarrollo de programas de ejercicio físi$\mathrm{CO}^{2}$. Sin embargo, ciertas limitaciones, como la reducida adherencia al programa, la dificultad para controlar la carga (volumen e intensidad) o la poca especificidad del programa, han impedido una reducción mayor de la mortandad. Debido a ello, se hace necesario definir nuevas estrategias o propuestas orientadas a la motivación, el control y el seguimiento de los programas de ejercicio físico en este colectivo. El ejercicio físico desarrollado a través de plataformas tecnológicas de realidad virtual puede reducir las limitaciones expuestas, si bien son necesarios más estudios que avalen estos resultados ${ }^{3}$. En los últimos años, los sistemas de realidad virtual han sido objeto de análisis y desarrollo en diferentes sectores, aplicándose esta tecnología en el gaming, la enseñanza y el entrenamiento físico y cognitivo. Debido a los buenos resultados que la realidad virtual ha generado en el entrenamiento físico, distintos centros y clínicas hospitalarias han desarrollado protocolos de realidad virtual que han incorporado en los procesos de rehabilitación $n^{4,5}$.

La realidad virtual implica una simulación basada en la estimulación sensorial controlada; es decir, es una simulación computarizada de espacios diversos en los que se puede interactuar con los objetos generados y explorarlos. En la actualidad, esta tecnología es muy aplicada con fines lúdicos (gaming) o de enseñanza, pero su uso es aún reducido en el ámbito médico ${ }^{5}$.

La incorporación de la realidad virtual a los programas terapéuticos basados en el ejercicio físico ofrece una nueva dimensión a la terapia convencional, ya que genera un entorno amable y motivante para el paciente durante el desarrollo de la terapia clínica. La revisión sistemática realizada por García Bravo, et al. ${ }^{6}$ señala que un programa de rehabilitación con soporte en realidad virtual aplicado a pacientes con enfermedades coronarias muestra resultados significativamente mejores que la aplicación del mismo programa de rehabilitación sin realidad virtual, lo cual genera, además, sentimientos de participación más positivos, tranquilidad durante el desarrollo de la sesión y entrenamientos más largos e intensos. En la actualidad, la cantidad de estudios científicos que emplean esta tecnología (realidad virtual) en el desarrollo de programas de rehabilitación cardiaca está en aumento; sin embargo, estos presentan una gran heterogeneidad en cuanto al programa desarrollado y la plataforma virtual empleada, lo que dificulta la generalización de conclusiones sobre la eficacia del tipo de programa y la plataforma empleada. Por lo anterior, esta investigación tuvo como objetivo efectuar una revisión sistemática sobre la eficacia de los programas de rehabilitación cardiaca desarrollados en diferentes plataformas de realidad virtual.

\section{Método}

\section{Registro}

Se siguieron las pautas de la guía Prefered Reporting Items for Systematic Reviews and Meta-Analyses (PRISMA) para el desarrollo de revisiones sistemáticas y metaanálisis?.

\section{Criterios de elegibilidad}

Se seleccionaron ensayos clínicos (aleatorizados o no aleatorizados) que evaluaran el efecto de protocolos de rehabilitación cardiaca desarrollados sobre plataformas de realidad virtual. La muestra de cada ensayo clínico debía estar compuesta por pacientes mayores de 18 años, con diagnóstico de enfermedad cardiovascular confirmada por un cardiólogo y con recomendación de desarrollo de un programa de rehabilitación cardiaca. Respecto al programa de rehabilitación cardiaca, este debía tener como mínimo una frecuencia 
de dos veces por semana y una duración de 3 meses.

\section{Fuente de información}

Se llevó a cabo una búsqueda bibliográfica en las bases de datos Scopus, Sport-Discus, PubMed (Medline), Web of Science y Dialnet. Esta búsqueda se basó en la aplicación de realidad virtual en protocolos de rehabilitación cardiaca o rehabilitación en general con el fin de abarcar todo el espectro de aplicación en las diferentes enfermedades. La búsqueda se hizo desde el 1 de diciembre de 2019 hasta el 16 de marzo de 2020. En caso de requerir ampliar alguna información, se intentó contactar con los autores. En la tabla 1 se pueden consultar todos los detalles de la estrategia de búsqueda (palabras clave, filtros aplicados, ecuaciones de búsqueda y resultados obtenidos).

\section{Selección de los estudios}

Los artículos identificados en cada una de las bases de datos fueron examinados por dos revisores de manera independiente; en el caso de haber alguna discrepancia se consultó a un tercer revisor. Se analizaron en un primer momento el título y el resumen (abstract) de cada documento seleccionado en las bases de datos. Los resultados obtenidos fueron consignados en una base de datos y los documentos se clasificaron en función de la enfermedad sobre la cual se desarrollaba el programa de ejercicio físico con realidad virtual. Se escogieron solo los artículos que trataban de "cardiac rehabilitation" y se descartaron los duplicados. Si la información era suficiente como para aplicar los criterios de selección, se procedía a clasificar el estudio en cuestión como «preválido» o «no válido». En los casos en los que el título y el resumen no fueron suficientes para realizar dicha clasificación, se realizó una lectura superficial del artículo en texto completo. Una vez identificados los artículos objeto de estudio, se realizó una lectura más detallada de los documentos seleccionados como «preválidos» para seguir aplicando los criterios de selección, descartando todos aquellos que no correspondían a ensayos clínicos. Los criterios de inclusión utilizados durante el proceso fueron los siguientes: 1) utilización de la realidad virtual en procesos de rehabilitación cardiaca; 2) artículos con acceso a texto completo (full text); y 3 ) ensayos clínicos. Por otra parte, los criterios de exclusión utilizados fueron los siguientes: 1) estudios que no tratasen un proceso de rehabilitación; 2) estudios sobre enfermedades que no
Tabla 1. Estrategia de búsqueda para realidad virtual y rehabilitación cardiaca

\begin{tabular}{|l|l|}
\hline Scopus & $\begin{array}{l}\text { Virtual AND Reality AND Cardiac } \\
\text { rehabilitation OR rehabilitation }\end{array}$ \\
\hline Palabras clave & Access type, 2018-2019 \\
\hline Filtros aplicados & $\begin{array}{l}\text { (TITLE-ABS-KEY (virtual AND reality AND } \\
\text { stroke AND rehabilitation) AND } \\
\text { búsqueda y } \\
\text { resultados }\end{array}$ \\
$\begin{array}{l}\text { TITLE-ABS-KEY (clinical AND trial)) AND } \\
\text { (LIMIT-TO (ACCESSTYPE (OA))) AND } \\
\text { (LIMIT-TO (PUBYEAR, 2019) OR LIMIT-TO } \\
\text { (PUBYEAR, 2018)) } \rightarrow \text { (39) }\end{array}$ \\
\hline
\end{tabular}

\section{Sport Discus}

Palabras clave

Virtual AND Reality AND Rehabilitation

Filtros aplicados

Virtual reality, full text, aplicar material equivalentes

Ecuación de

búsqueda y

resultados

(Virtual AND Reality AND Rehabilitation) AND (LIMIT-TO (TEXT COMPLETE)) AND (LIMIT-TO (APPLY EQUIVALENT MATTERS)) AND (LIMIT-TO (MATTERS VIRTUAL REALITY)) $\rightarrow$ (28)

\section{PubMed (Medline)}

\begin{tabular}{|c|c|}
\hline Palabras clave & Virtual AND Reality AND Rehabilitation \\
\hline Filtros aplicados & Clinical trial, last 5 years, full text \\
\hline $\begin{array}{l}\text { Ecuación de } \\
\text { búsqueda y } \\
\text { resultados }\end{array}$ & $\begin{array}{l}\text { ((“virtual reality"[MeSH Terms] OR } \\
\text { (“virtual"[All Fields] AND “reality"[All } \\
\text { Fields]) OR "virtual reality"[All Fields]) } \\
\text { AND ("rehabilitation"[Subheading] OR } \\
\text { "rehabilitation"[All Fields] OR } \\
\text { "rehabilitation"[MeSH Terms])) AND } \\
\text { (Clinical Trial[ptyp] AND “loattrfree full } \\
\text { text"[sb] AND “2014/12/16"[PDat] : } \\
\text { "2019/12/14"[PDat]) } \rightarrow \text { (97) }\end{array}$ \\
\hline
\end{tabular}

\section{Web of Science}

Palabras clave

Virtual AND Reality AND rehabilitation

Filtros aplicados

Virtual reality, 2018

Ecuación de

búsqueda $y$

(Virtual AND Reality AND Rehabilitation) resultados AND (LIMIT-TO (PUBLIC IN 2018-2019)) AND (LIMIT TO((CATEGORY REHABILITATION)) $\rightarrow$ (89)

\begin{tabular}{l|l|}
\hline Dialnet & \\
\hline Palabras clave & Virtual AND Reality AND rehabilitation \\
\hline Filtros aplicados & Acceso a texto completo \\
\hline $\begin{array}{l}\text { Ecuación de } \\
\text { búsqueda y } \\
\text { resultados }\end{array}$ & $\begin{array}{l}\text { (Virtual AND Reality AND Rehabilitation) } \\
\text { AND (LIMIT-TO (TEXT COMPLETE)) } \rightarrow \text { (27) }\end{array}$ \\
\hline
\end{tabular}

fueran cardiacas; 3) estudios repetidos en bases de datos; y 4) estudios carentes de ensayos clínicos. Finalmente, se realizó una retrobúsqueda a partir de la 
bibliografía de los resultados «válidos» para lograr una selección más completa.

\section{Proceso de extracción de los datos}

De los estudios escogidos se evaluaron las siguientes variables: aleatorización, tipo de realidad virtual, estudio pre-post/post, enfermedad cardiaca, variables de control, intervención, protocolo y resultados ${ }^{8}$.

\section{Evaluación de la calidad}

La calidad metodológica de los estudios fue evaluada independientemente y a ciegas por dos revisores $(B A, J C)$. Las evidencias sugieren que las mejores valoraciones y la consistencia de la evaluación de la calidad metodológica de los estudios se produce cuando los revisores no conocen los autores, el título, la revista ni la institución ${ }^{9}$. Cada documento fue evaluado usando los 11 ítems de la Escala de PEDro. Esta escala fue desarrollada para evaluar la calidad metodológica (proceso aleatorio de los estudios, técnica de cegamiento o proceso de análisis de los datos) en la literatura fisioterapéutica, y permite evaluar una gran variedad de diseños metodológicos. La puntuación de la escala abarca un rango de 0 a 10, y cuanto más alta sea la puntuación, mayor calidad metodológica tiene el estudio ${ }^{10}$.

\section{Análisis}

El análisis de los estudios seleccionados mostró una gran heterogeneidad en los sistemas de realidad virtual, así como en las variables empleadas para determinar el efecto de los programas de rehabilitación cardiaca aplicados a través de la realidad virtual. Esta heterogeneidad ha imposibilitado desarrollar un análisis de tipo estadístico o metaanálisis. Por consiguiente, la información fue analizada de manera cualitativa, teniendo en cuenta las características metodológicas de los estudios y los resultados reportados en cada uno de ellos.

\section{Resultados}

\section{Selección de los estudios}

La búsqueda en las diferentes bases de datos generó 280 registros. De ellos, 249 fueron descartados en la primera selección por no tratar enfermedades de origen cardiaco, de modo que la selección quedó en 31 registros. Se descartaron siete por tratarse de duplicados, tres por no contener el texto completo, cuatro por no ajustarse al tema de estudio y cuatro por el tipo de publicación, con lo que quedó un total de 13 registros. Se realizó una lectura completa de estos 13 artículos, de los cuales se excluyeron cuatro por no ser ensayos clínicos y tres por carecer de grupo de control. Finalmente, se obtuvieron seis artículos, a los que se sumaron dos tras realizar un retrobúsqueda de los ensayos seleccionados, con un resultado final de ocho ensayos a estudiar (Fig. 1).

\section{Características de los estudios}

Se analizaron ocho ensayos clínicos ${ }^{11-18}$, seis de los cuales tenían un diseño aleatorizado controlado ${ }^{11-14,16,18}$. Respecto a los sistemas de realidad virtual empleados, cinco estudios emplearon videoconsolas, como Nintendo $\mathrm{Wii}^{14-16}$ o Microsoft Kinect ${ }^{17,18}$, y dos estudios emplearon sistemas virtuales creados específicamente para el desarrollo del estudio ${ }^{12,13}$; el estudio de Cacau, et al. ${ }^{11}$ no especifica el tipo de realidad virtual empleado. En cuanto a los momentos de análisis, cinco ensayos clínicos presentan valores de sus indicadores en los momentos preintervención y posintervención ${ }^{11,14,15,17,18}$. Los estudios restantes solo presentaron valores del momento posintervención (Tabla 2).

\section{Calidad de los estudios}

La calidad metodológica se evaluó siguiendo los ítems de la Escala PEDro con base en las necesidades de evaluación de los objetivos del estudio, y en un rango de 1 a 10 puntos. Del total de los artículos analizados, cinco obtuvieron un resultado de 6 pun$\operatorname{tos}^{11-13,16,18}$, mientras que, de los restantes, uno obtuvo 5 puntos $^{17}$ y dos obtuvieron 4 puntos ${ }^{14,15}$. Cabe destacar que en todos los artículos se especificaron los criterios de selección de la muestra, siendo la selección oculta en siete ensayos clínicos ${ }^{11-14,16-18}$. Ninguna de las terapias aplicadas fue cegada y solamente en un artículo se presentaron los resultados de todos los sujetos que recibieron el tratamiento ${ }^{15}$. Con base en ello, podemos afirmar que la calidad metodológica de los estudios analizados no parece estar condicionada por el año de publicación, y que los estudios presentan una calidad baja (Tabla 3).

\section{Descripción de los estudios}

La información recopilada en los diferentes estudios reflejó un tamaño de muestra total de 872 sujetos, con 
Rev Colomb Cardiol. 2021;28(6)

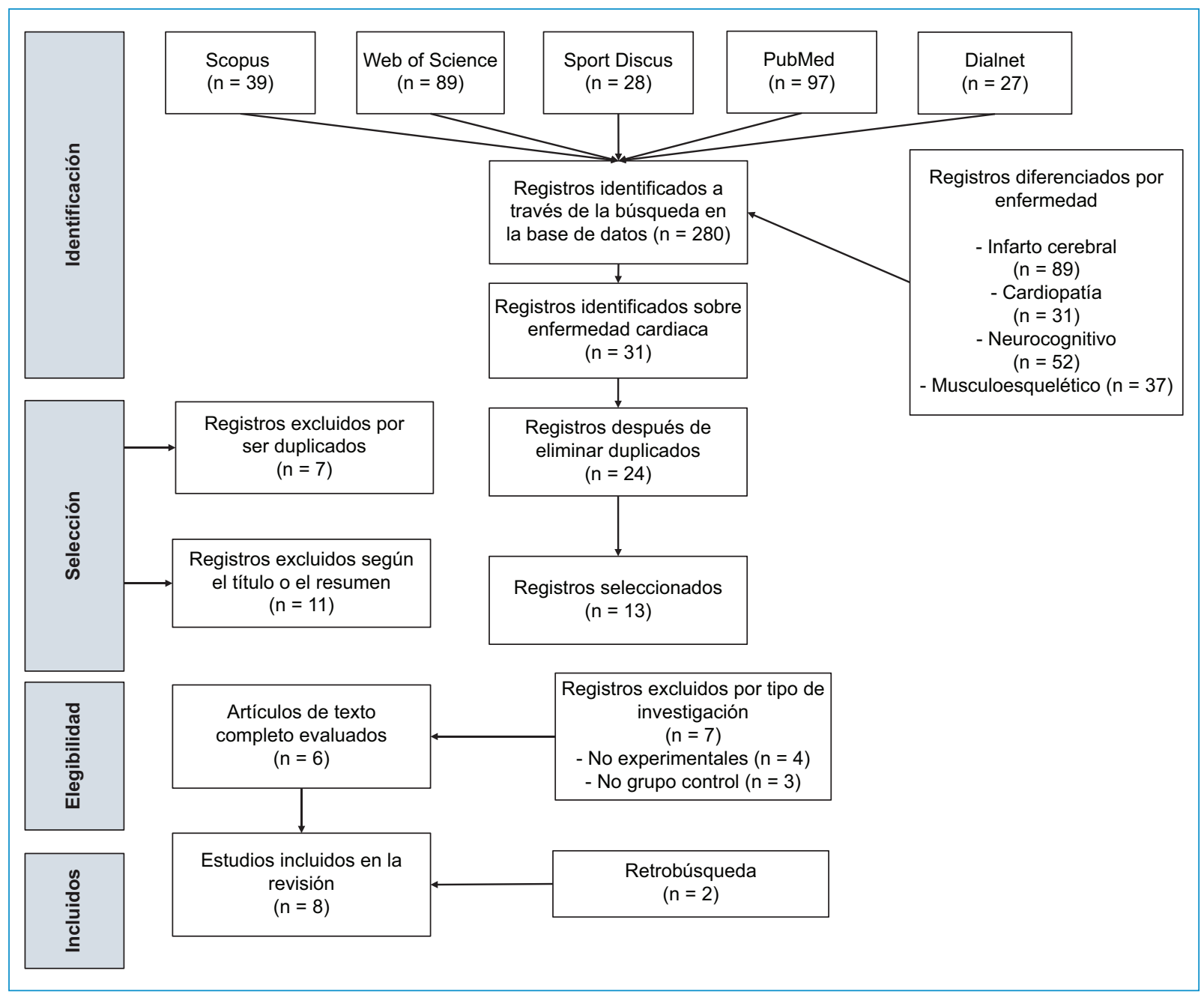

Figura 1. Diagrama de flujo de la selección de artículos (PRISMA).

Tabla 2. Tabla resumen de los estudios seleccionados

\begin{tabular}{|l|l|l|l|}
\hline Autor & Aleatorizado & Tipo de realidad virtual & $\begin{array}{l}\text { Estudios } \\
\text { pre-post/post }\end{array}$ \\
\hline Cacau, et al. (2013) & Sí & Sistema de realidad virtual no especificado & Pre-post \\
\hline Chuang, et al. (2005) & Sí & Telepresence Cardiac Rehabilitation Program & Post \\
\hline Chuang, et al. (2006) & Sí & Telepresence Cardiac Rehabilitation Program & Post \\
\hline Jaarsma, et al. (2015) & Sí & Nintendo Wii & Pre-post \\
\hline Klompstra, et al. (2014) & No & Nintendo Wii & Pre-post \\
\hline Ruivo, et al. (2017) & Sí & Nintendo Wii & Post \\
\hline Vieria, et al. (2017) $)^{17}$ & No & Microsoft Kinect-Project Kinect-RehabPlay & Pre-post \\
\hline Vieira, et al. (2017) ${ }^{18}$ & Sí & Microsoft Kinect-Project Kinect-RehabPlay & Pre-post \\
\hline
\end{tabular}


Tabla 3. Evaluación de la calidad metodológica de los estudios

\begin{tabular}{|c|c|c|c|c|c|c|c|c|}
\hline Indicadores & $\begin{array}{l}\text { Cacau, } \\
\text { et al. } \\
(2013)^{11}\end{array}$ & $\begin{array}{l}\text { Chuang, } \\
\text { et al. } \\
(2005)^{12}\end{array}$ & $\begin{array}{l}\text { Chuang, } \\
\text { et al. } \\
(2006)^{13}\end{array}$ & $\begin{array}{l}\text { Jaarsma, } \\
\text { et al. } \\
(2015)^{14}\end{array}$ & $\begin{array}{l}\text { Klompstra, } \\
\text { et al. } \\
(2014)^{15}\end{array}$ & $\begin{array}{l}\text { Ruivo, } \\
\text { et al. } \\
(2017)^{16}\end{array}$ & $\begin{array}{l}\text { Vieira, } \\
\text { et al. } \\
(2017 \mathrm{a})^{17}\end{array}$ & $\begin{array}{c}\text { Vieira, et al. } \\
(2017 b)^{18}\end{array}$ \\
\hline $\begin{array}{l}\text { 1. Los criterios de } \\
\text { elección fueron } \\
\text { especificados } \\
\text { (no puntuable) }\end{array}$ & 1 & 1 & 1 & 1 & 1 & 1 & 1 & 1 \\
\hline $\begin{array}{l}\text { 2. Los sujetos fueron } \\
\text { asignados al azar a } \\
\text { los grupos }\end{array}$ & 1 & 1 & 1 & 1 & 0 & 1 & 0 & 1 \\
\hline $\begin{array}{l}\text { 3. La asignación } \\
\text { fue oculta }\end{array}$ & 1 & 1 & 1 & 1 & 0 & 1 & 1 & 1 \\
\hline $\begin{array}{l}\text { 4. Los grupos } \\
\text { fueron similares } \\
\text { al inicio en } \\
\text { relación a los } \\
\text { indicadores de } \\
\text { pronóstico más } \\
\text { importantes }\end{array}$ & 1 & 1 & 1 & 1 & 1 & 1 & 1 & 1 \\
\hline $\begin{array}{l}\text { 5. Todos los } \\
\text { sujetos fueron } \\
\text { cegados }\end{array}$ & 1 & 1 & 1 & 1 & 0 & 1 & 1 & 1 \\
\hline $\begin{array}{l}\text { 6. Todos los } \\
\text { terapeutas que } \\
\text { administraron la } \\
\text { terapia fueron } \\
\text { cegados }\end{array}$ & 0 & 0 & 0 & 0 & 0 & 0 & 0 & 0 \\
\hline $\begin{array}{l}\text { 7. Todos los } \\
\text { evaluadores } \\
\text { que midieron al } \\
\text { menos un } \\
\text { resultado clave } \\
\text { fueron cegados }\end{array}$ & 0 & 0 & 0 & 0 & 0 & 0 & 0 & 0 \\
\hline $\begin{array}{l}\text { 8. Las medidas de al } \\
\text { menos uno de los } \\
\text { resultados clave } \\
\text { fueron obtenidas } \\
\text { de más del } 85 \% \\
\text { de los sujetos } \\
\text { inicialmente } \\
\text { asignados a los } \\
\text { grupos }\end{array}$ & 0 & 0 & 0 & 0 & 0 & 0 & 0 & 0 \\
\hline $\begin{array}{l}\text { 9. Se presentaron } \\
\text { resultados de } \\
\text { todos los sujetos } \\
\text { que recibieron } \\
\text { tratamiento }\end{array}$ & 0 & 0 & 0 & 0 & 1 & 0 & 0 & 0 \\
\hline $\begin{array}{l}\text { 10. Los resultados de } \\
\text { comparaciones } \\
\text { estadísticas entre } \\
\text { grupos fueron } \\
\text { informados para } \\
\text { al menos un } \\
\text { resultado clave }\end{array}$ & 1 & 1 & 1 & 0 & 1 & 1 & 1 & 1 \\
\hline $\begin{array}{l}\text { 11. El estudio } \\
\text { proporciona } \\
\text { medidas } \\
\text { puntuales y de } \\
\text { variabilidad para } \\
\text { al menos un } \\
\text { resultado clave }\end{array}$ & 1 & 1 & 1 & 0 & 1 & 1 & 1 & 1 \\
\hline Score total & 6 & 6 & 6 & 4 & 4 & 6 & 5 & 6 \\
\hline
\end{tabular}


un rango de edad entre 49,20 y 68,67 años ${ }^{11,12}$. El tamaño de la muestra por estudio fue muy variable; así, un estudio incluyó 600 sujetos $^{14}$, mientras que en otro solo hubo $24^{13}$. En cuanto a los indicadores empleados para comprobar el efecto de los programas de rehabilitación cardiaca, es preciso indicar que son muy variados y que existen indicadores físicos y cognitivos. En tal sentido, cabe destacar que dos estudios incluyeron el análisis de la frecuencia cardiaca y el análisis del consumo de oxígeno ${ }^{12,13}$, cinco la capacidad aeróbica o capacidad de ejercicio de los sujetos evaluada mediante la FIM (Functional Independence Measure), el 6MWT (Six-Minute Walk Test) o cuestionarios como el MACNew questionaire ${ }^{11-15}$, tres el índice de masa corporal (IMC) $)^{13,15,17}$, tres los niveles de ansiedad y depresión ${ }^{14,15,18}$, dos la calidad de vida ${ }^{14,18}$, dos la motivación hacia el ejercicio ${ }^{14,15}$, uno la adherencia al programa $^{16}$, uno la duración de la estancia en el hospital $^{11}$ y otro la percepción de la salud ${ }^{11}$. Pese a que las enfermedades analizadas en los ocho artículos son de origen cardiaco, las especificaciones son distintas entre ellos. De esta manera, se obtuvieron enfermedades de cirugía poscardiaca ${ }^{11-13}$, insuficiencia cardia$\mathrm{ca}^{14,15}$, riesgo cardiovascular bajo-moderado ${ }^{16} \mathrm{y}$ enfermedad coronaria estable ${ }^{17,18}$ (Tabla 4).

\section{Discusión}

Esta revisión sistemática se llevó a cabo para verificar la efectividad del empleo de los sistemas de realidad virtual en los protocolos de rehabilitación cardiaca centrando el análisis en la adherencia del paciente al programa, así como en los resultados generados por los protocolos tanto en variables físicas (capacidad aeróbica, potencia aeróbica e IMC) como en variables cognitivas (ansiedad y depresión). Con base en los resultados obtenidos en los diferentes ensayos clínicos, se determinó que el uso de los sistemas de realidad virtual en los protocolos de rehabilitación cardiaca provoca un aumento de la adherencia a los programas y genera mayores mejoras en las variables físicas que aquellos protocolos que no emplean estos sistemas.

Si bien están claros los beneficios que generan los programas de rehabilitación cardiaca en la salud de los pacientes cardiópatas, se destaca la falta de adherencia de los pacientes a estos programas. En los primeros 3 a 6 meses se observa un abandono progresivo del programa de rehabilitación cardiaca. Es a partir del sexto mes cuando este abandono se hace más acentuado, y es ahí cuando el porcentaje de adherencia al programa está entre el $30 \%$ y el $60 \%$ de los inscritos inicialmente ${ }^{19}$. Por ello, el uso de sistemas tecnológicos novedosos con protocolos de rehabilitación convencionales adquiere gran importancia, pues puede aumentar la adherencia a los programas de rehabilitación. Los sistemas de realidad virtual y los videojuegos son herramientas que se caracterizan por fomentar la realización de ejercicio físico de una forma más entretenida y agradable 6 . La aplicación activa de sistemas de realidad virtual en pacientes que hayan pasado por una enfermedad cardiovascular pasa a ser una estrategia prioritaria, ya que puede aumentar el interés del paciente y, con ello, la adherencia. Ruivo, et al. ${ }^{16}$ evaluaron la tasa de adherencia en un programa de rehabilitación cardiaca con el uso de un videojuego de realidad virtual (Nintendo Wii). Las sesiones realizadas mediante este soporte generaron una tasa de adherencia al programa del $68 \%$, superior al reflejado en programas sin soporte virtual ${ }^{19}$. Además, este ensayo clínico ${ }^{16}$ reveló una disposición más activa de los participantes en el desarrollo del programa, quizás debido a que la plataforma empleada era sencilla, asequible y adaptable para cada paciente.

De forma global, es posible indicar que los resultados obtenidos en los diferentes ensayos clínicos analizados en este estudio señalan que la aplicación de programas de rehabilitación cardiaca con soporte de realidad virtual generan una mejoras sustanciales en cuanto a capacidad aeróbica, potencia aeróbica, capacidad funcional e IMC, en comparación con los programas de terapia convencional.

La capacidad y la potencia aeróbica se vieron especialmente influenciadas por los sistemas de realidad virtual, generándose una aceleración de la recuperación máxima de la función cardiovascular de los pacientes $^{12-15}$. Chuang, et al..$^{13}$ explican en su estudio que los pacientes alcanzaron velocidades más altas en comparación con los sujetos del grupo de control (sin realidad virtual). La causa puede deberse a que la experiencia con realidad virtual genera una inmersión del paciente en el sistema, disminuyendo la conciencia sobre las incomodidades físicas que aparecen durante la realización del programa, lo que hace que este pueda ejercitarse a un nivel más alto en relación con sus capacidades reales y no verse sesgado por su propia influencia.

Jaarsma, et al..$^{14}$ y Klompstra, et al..$^{15}$ evaluaron el efecto de los sistemas de realidad virtual sobre la capacidad muscular y el nivel de actividad física, y observaron que los pacientes que usaron sistemas de realidad virtual alcanzaron valores más elevados en estas variables frente a los que no la usaron. 


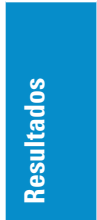

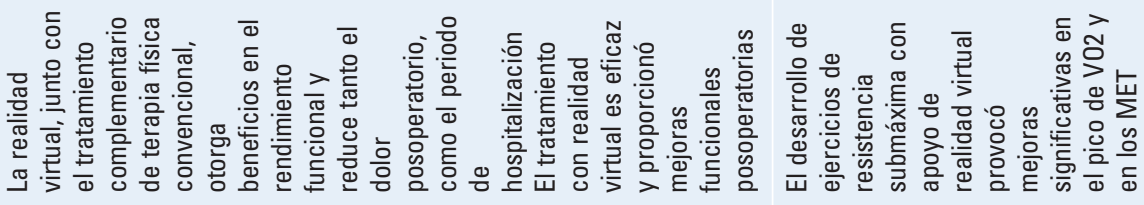

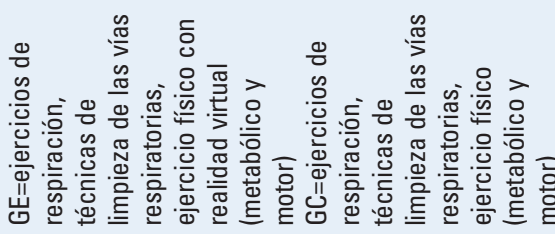

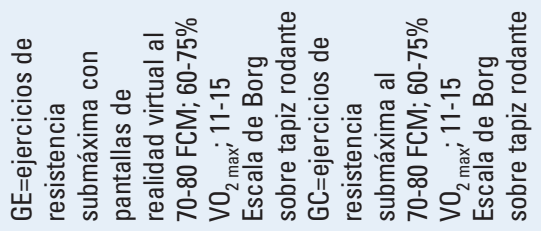

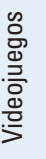

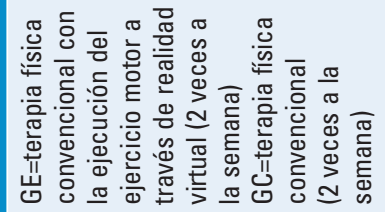

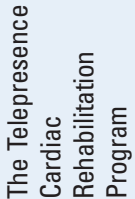
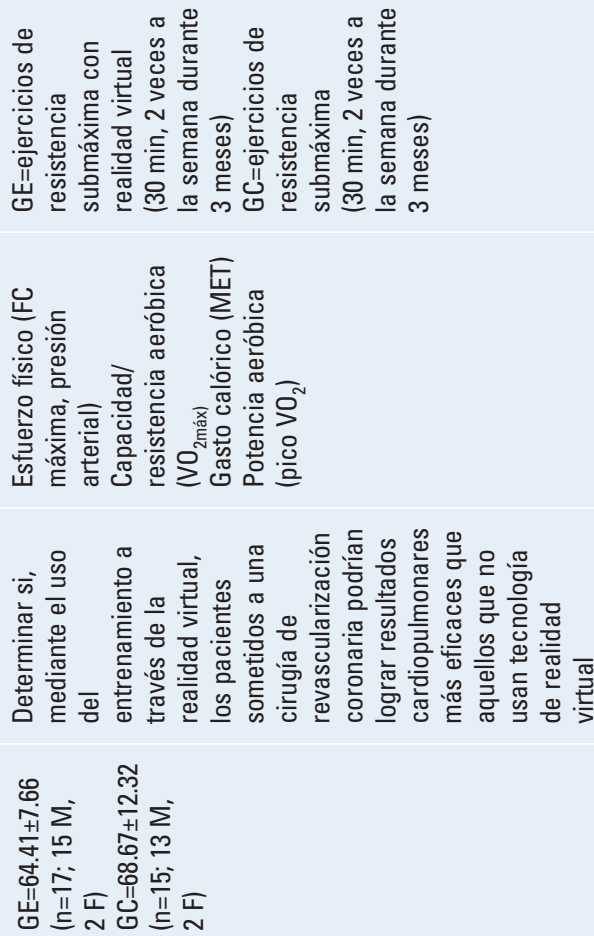

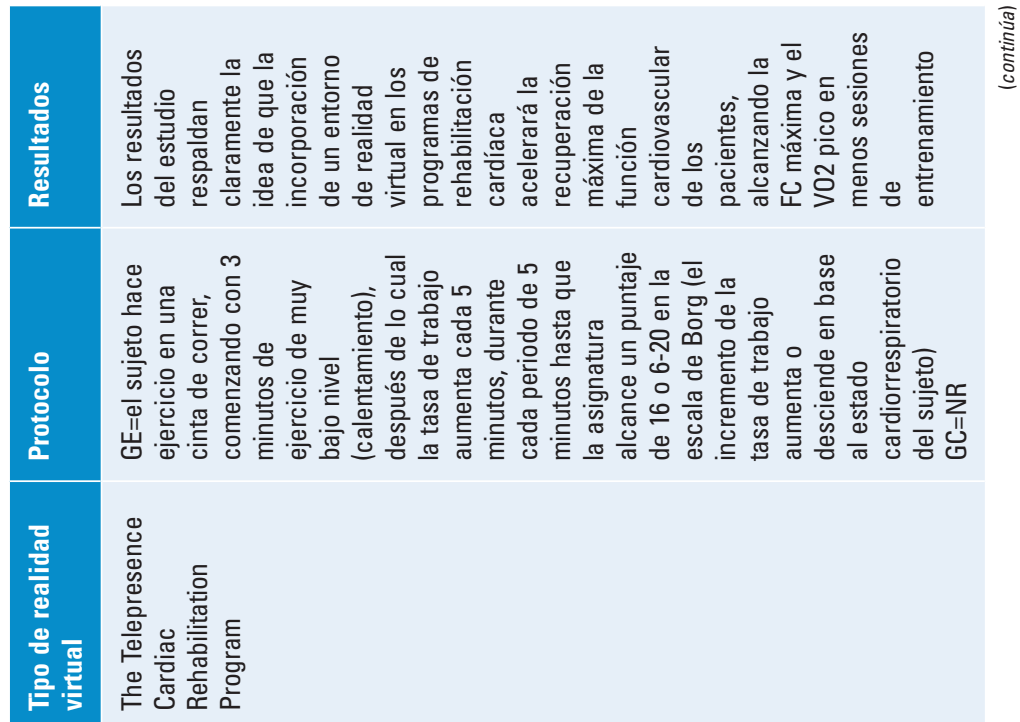

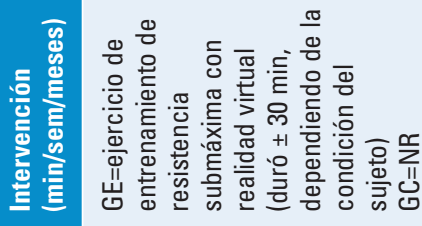

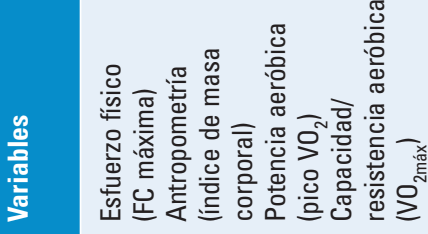

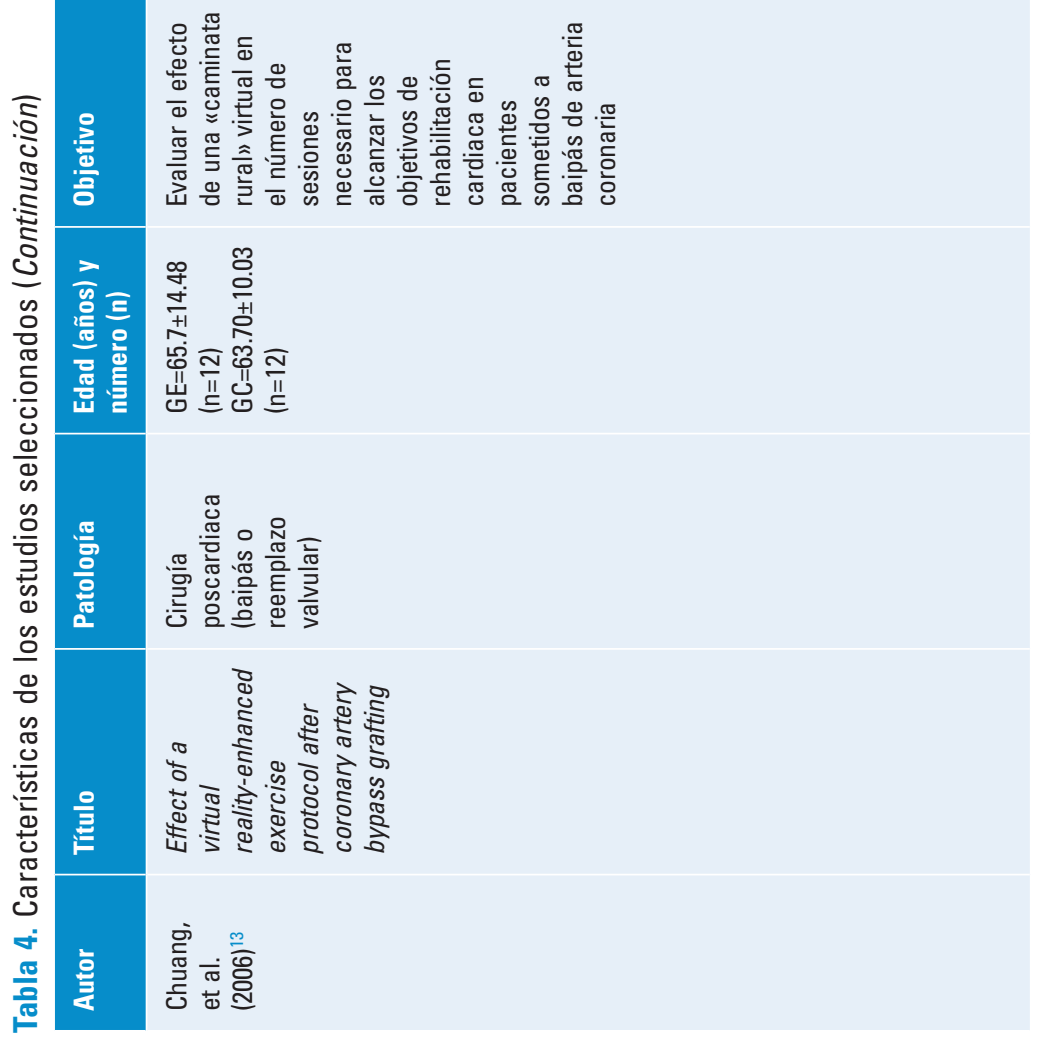




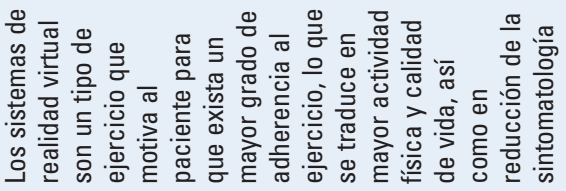

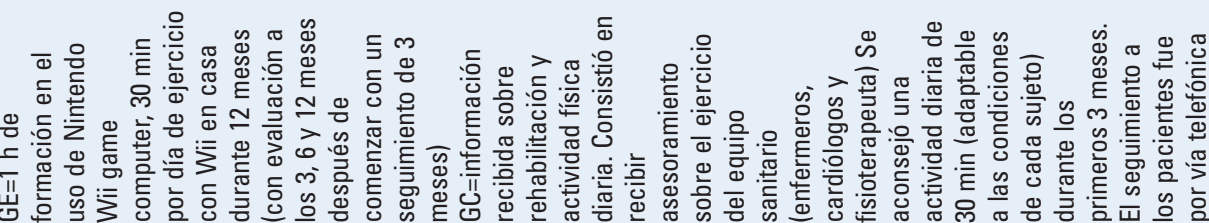

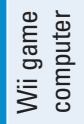

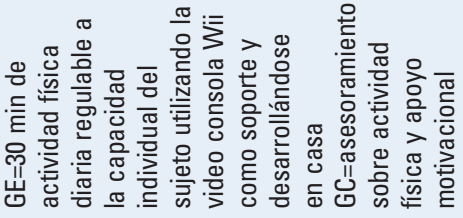

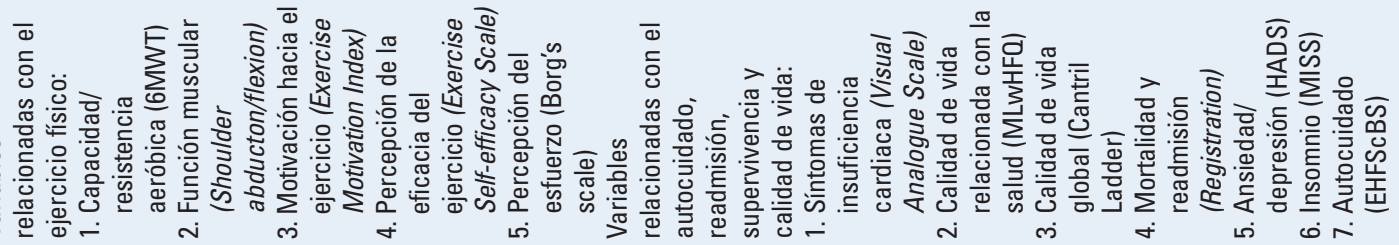

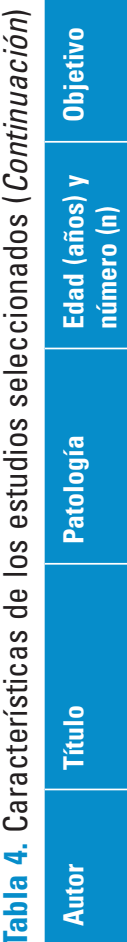

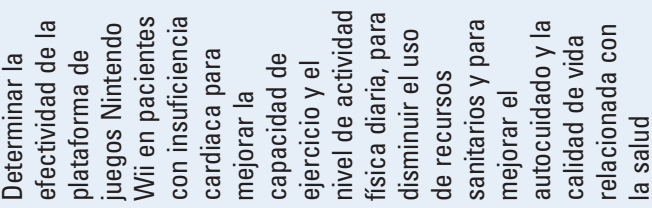

승

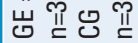

$\frac{-\pi}{4}$

$\frac{\bar{\omega}}{0}$.

穿

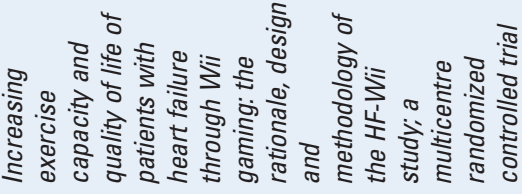

焉 


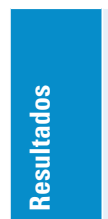

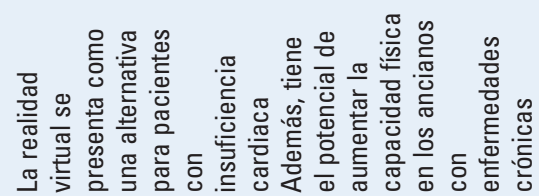

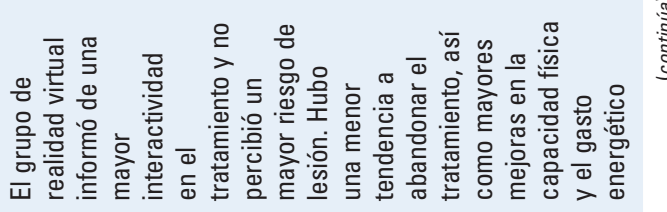

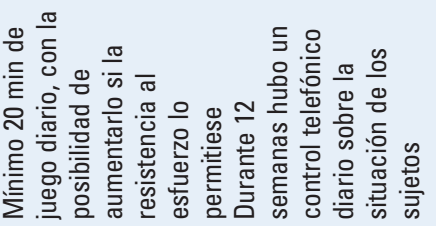

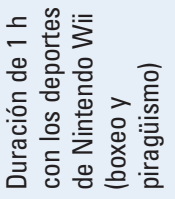

覀

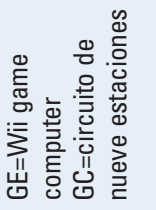

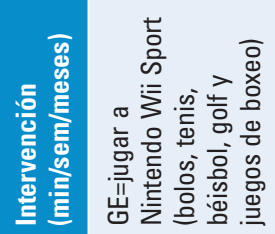

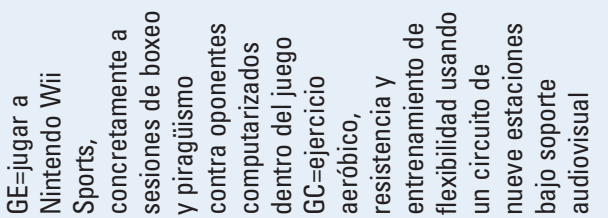

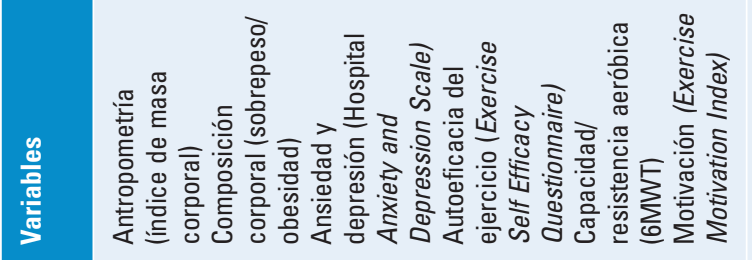
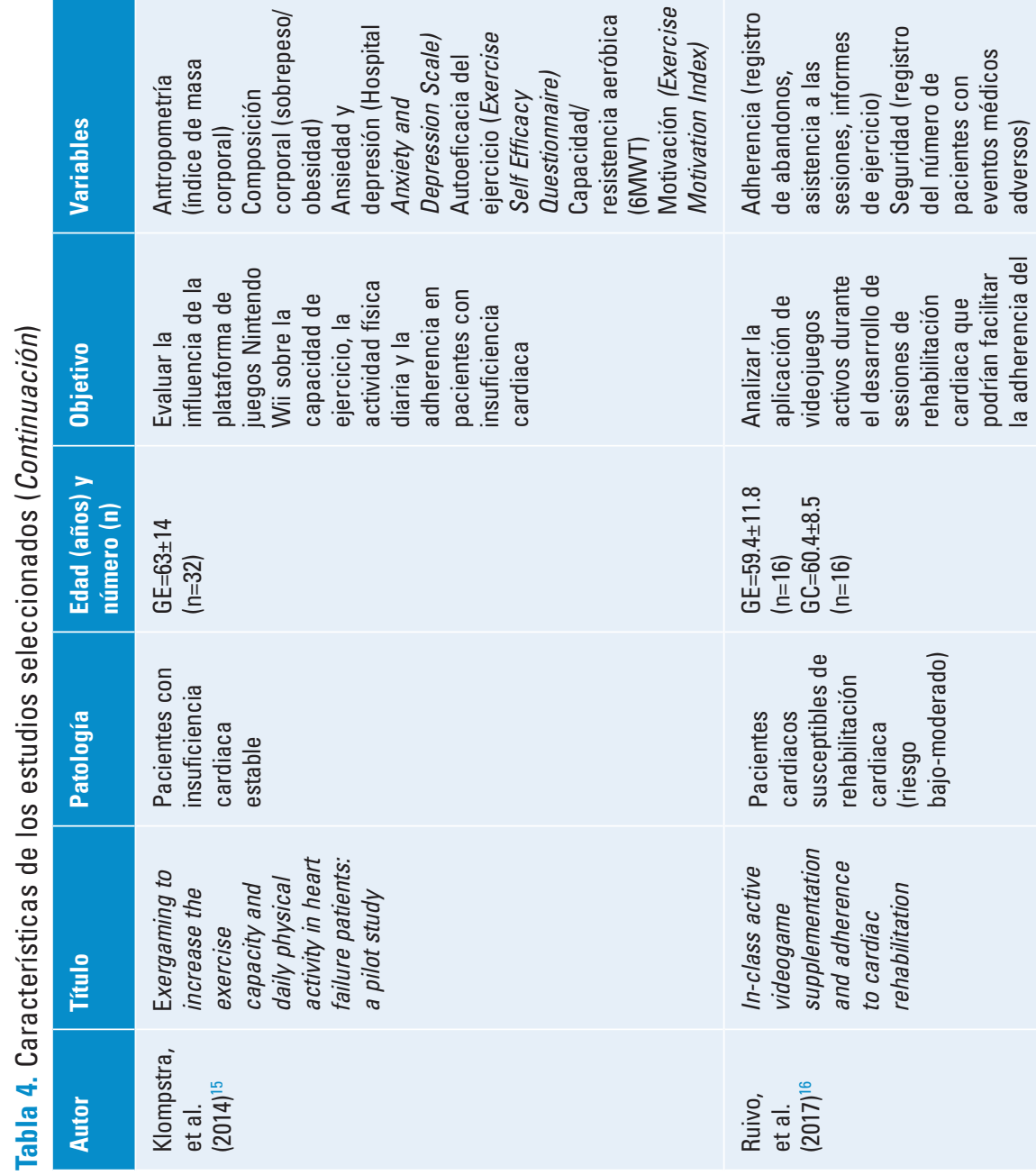

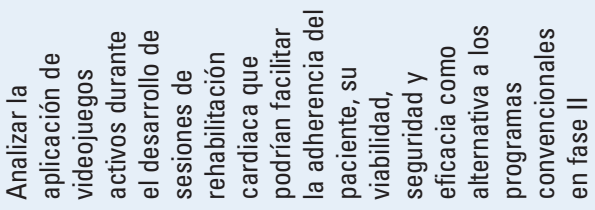
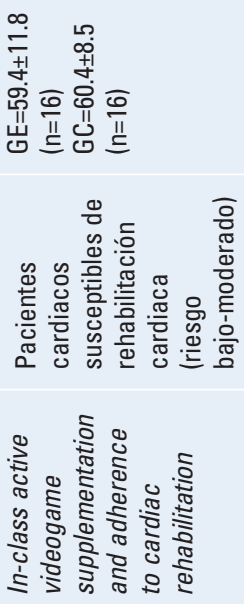


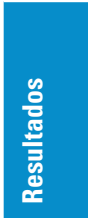

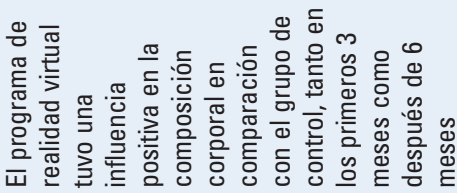

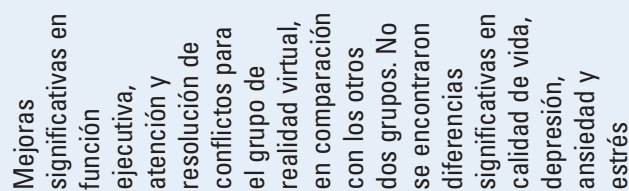

产

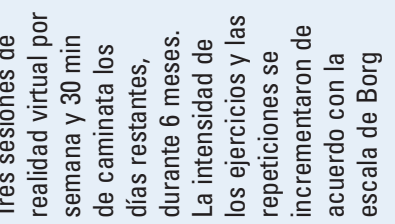

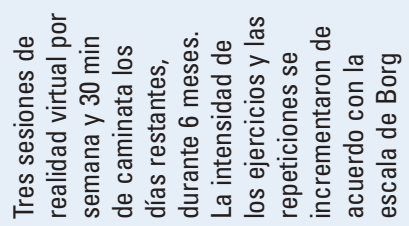

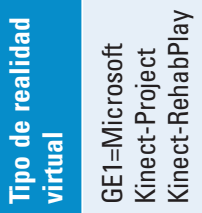

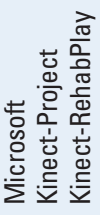

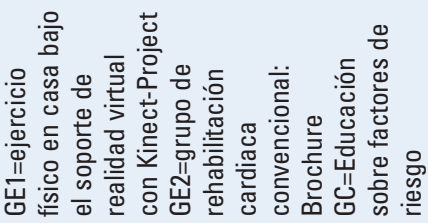

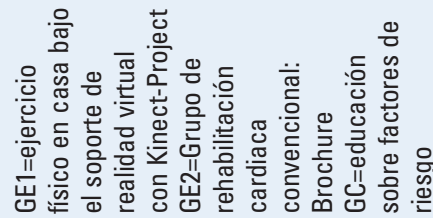

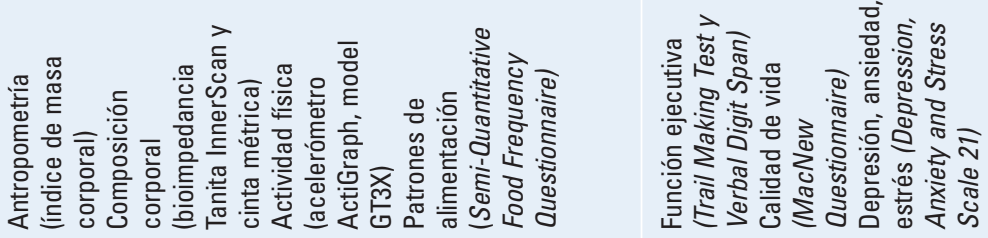

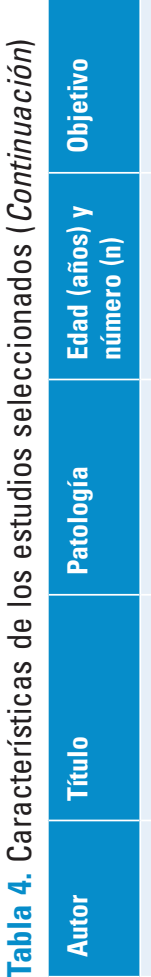

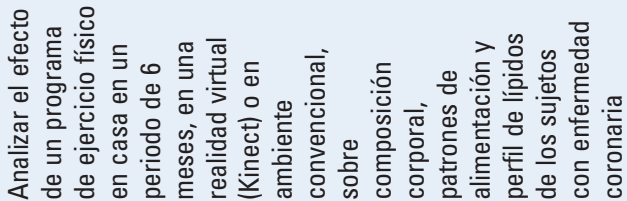

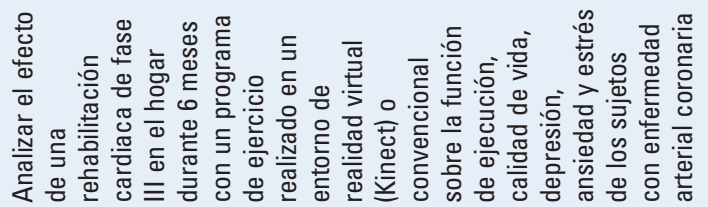

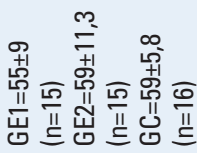

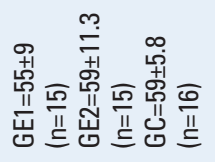

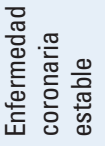

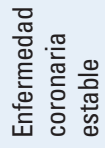

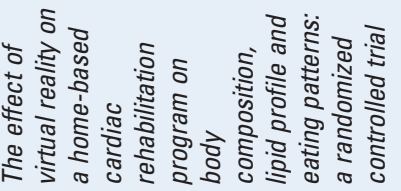

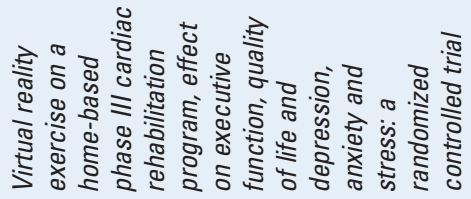

高离言言

要

نं

\$ 
EI IMC es un parámetro que permite estimar la cantidad de grasa corporal de un paciente. Tiene una relación directa con un mayor riesgo de padecer una enfermedad cardiovascular, y por ello, uno de los objetivos principales de cualquier programa de rehabilitación cardiaca debe ser controlar los valores de este parámetro. Todos los programas de rehabilitación cardiaca deben estar compuestos por ejercicios de resistencia aeróbica, de manera que produzcan un efecto notable en la pérdida de grasa corporal y en la ganancia de masa magra ${ }^{22}$. Vieira, et al. ${ }^{17}$ y Klompstra, et al. ${ }^{15}$ estudiaron la eficacia de programas de ejercicio físico bajo sistemas de realidad virtual en pacientes con enfermedad cardiaca. Ambos autores concluyen que se produjeron diferencias significativas entre el grupo que usaba el sistema de realidad virtual y el que no lo usaba, pues los primeros redujeron su IMC. Esta diferencia se debió, según los autores, al aumento de la intensidad de trabajo del grupo que desarrolló el programa sobre un sistema de realidad virtual, debido a la atracción y a las sensaciones novedosas que generó el sistema sobre los pacientes.

En cuanto a las variables psicológicas (ansiedad y depresión) incluidas en los ensayos clínicos analiza$\operatorname{dos}^{14,15,18}$, es preciso indicar que los resultados obtenidos no son concluyentes. Los sistemas de realidad virtual parecen no influir en los niveles de ansiedad y depresión en comparación con aquellos que no usan la realidad virtual.

Por otro lado, se observaron diferentes limitaciones durante el desarrollo de esta revisión. En general, la principal es la baja calidad de los ensayos clínicos sobre rehabilitación cardiaca con soporte de realidad virtual, lo cual ha imposibilitado el desarrollo de un metaanálisis. Pese a que la revisión está compuesta por seis ensayos clínicos aleatorizados controla$\operatorname{dos}^{11-14,16,18}$, la información aportada en cada uno de ellos no ha sido suficiente como para poder establecer conclusiones de mayor rigor científico. Se encontraron estudios que no reportan de manera cuantitativa sus resultados $^{14}$, uno carece de grupo de control ${ }^{15}$ y otro solo evalúa la adherencia al sistema de realidad virtual ${ }^{16}$, pese a que recogen variables de interés para el estudio (frecuencia cardiaca e IMC). Otra limitación a destacar en este estudio es el empleo de diferentes sistemas de realidad virtual en los ensayos clínicos analizados; así, el sistema de realidad virtual utilizado en cinco ensayos clínicos ${ }^{14-18}$ ha sido un sistema de estimulación sensorial basada en videojuegos, en otros tres el sistema de realidad virtual empleado ha sido un programa específico de telepresencia ${ }^{12,13}$, y en un ensayo clínico no se especifica el sistema empleado ${ }^{11}$, lo que ha dificultado establecer conclusiones según el sistema de realidad virtual empleado.

\section{Conclusiones}

De acuerdo con los resultados de este estudio, se puede afirmar que la incorporación de los sistemas de realidad virtual a los programas de rehabilitación cardiaca convencional aumenta la adherencia, genera mejoras en la capacidad y la potencia aeróbica, y reduce el IMC; cambios que son mayores que los logrados mediante los programas desarrollados sin realidad virtual.

\section{Financiamiento}

Los autores declaran no haber recibido ningún tipo de financiamiento para el desarrollo de esta investigación.

\section{Conflicto de intereses}

Los autores declaran no tener conflictos de intereses.

\section{Responsabilidades éticas}

Protección de personas y animales. Los autores declaran que para esta investigación no se han realizado experimentos en seres humanos ni en animales.

Confidencialidad de los datos. Los autores declaran que en este artículo no aparecen datos de pacientes.

Derecho a la privacidad y consentimiento informado. Los autores declaran que en este artículo no aparecen datos de pacientes.

\section{Bibliografía}

1. Maroto Montero JM, Zarzosa C. Rehabilitación cardiovascular. Madrid: Médica Panamericana; 2011.

2. Anderson L, Oldridge N, Thompson DR, Zwisler AD, Rees K, Martin N et al. Exercise-based cardiac rehabilitation for coronary heart disease Cochrane systematic review and meta-analysis. J Am Coll Cardiol. 2016:67:1-12

3. Saposnik G, Levin M. Virtual reality in stroke rehabilitation: a meta-analysis and implications for clinicians. Stroke. 2011;42:1380-6.

4. Miller KJ, Adair BS, Pearce AJ, Said CM, Ozanne E, Morris MM. Effectiveness and feasibility of virtual reality and gaming system use at home by older adults for enabling physical activity to improve health-related domains: a systematic review. Age Ageing. 2014;43:188-95.

5. Borrego A, Latorre J, Alcañiz M, Llorens R. Comparison of oculus rift and HTC Vive: feasibility for virtual reality-based exploration, navigation, exergaming, and rehabilitation. Games Health J. 2018;7:151-6.

6. García-Bravo S, Cuesta-Gómez A, Campuzano-Ruiz $R$ López-Navas MJ, Domínguez-Paniagua J, Araújo-Narváez A, et al. Virtual reality and video games in cardiac rehabilitation programs. A systematic review. Disabil Rehabil. 2021;43:448-57. 
7. Urrútia G, Bonfill X. Declaración PRISMA: una propuesta para mejorar la publicación de revisiones sistemáticas y metaanálisis. Med Clin (Barc) 2010;135:507-11.

8. Higgins JPT, Green S. Manual Cochrane de revisiones sistemáticas de intervención. Cochrane; 2011. p. 1-639. Disponible en: chrome-extension://oemmndcbldboiebfnladdacbdfmadadm/https://es.cochrane.org/sites/es.cochrane.org/files/public/uploads/Manual Cochrane 510 reduit.pdf.

9. Jadad AR, Moore RA, Carroll D, Jenkinson C, Reynolds DJ, Gavaghan DJ, et al. Assessing the quality of reports of randomized clinical trials: is blinding necessary? Control Clin Trials. 1996;17:1-12.

10. Maher CG, Sherrington C, Herbert RD, Moseley AM, Elkins M. Reliability of the PEDro Scale for rating quality of randomized controlled trials. Phys Ther. 2003;83:713-21.

11. Cacau LA, Oliveira GU, Maynard LG, de Araújo Filho AA, da Silva Junior WM, Cerqueria Neto ML, et al. O uso da realidade virtual como ferramenta complementar no pós-operatório de cirurgia cardíaca. Braz J Cardiovasc Surg. 2013;28:281-9.

12. Chuang TY, Sung WH, Lin CY. Application of a virtual reality-enhanced exercise protocol in patients after coronary bypass. Arch Phys Med Rehabil. 2005;86:1929-32.

13. Chuang TY, Sung WH, Chang HA, Wang RY. Effect of a virtual reality-enhanced exercise protocol after coronary artery bypass grafting. Phys Ther. 2006;86:1369-77.
14. Jaarsma T, Klompstra L, Gal T, Ben Boyne J, Bäck M, Dickstein K et al. Increasing exercise capacity and quality of life of patients with heart failure through Wii gaming: the rationale, design and methodology of the HF-Wii study: a multicentre randomized controlled trial. Eur J Heart Fail. 2015;17:743-8.

15. Klompstra L, Jaarsma $T$, Strömberg A. Exergaming to increase the exercise capacity and daily physical activity in heart failure patients: a pilot study. BMC Geriatr. 2014;14:119.

16. Ruivo JM, Karim K, O'Shea R, Oliveira RC, Keary L, O'Brien C, et al. In-class active video game supplementation and adherence to cardiac rehabilitation. J Cardiopulm Rehabil Prev. 2017;37:274-8.

17. Vieira AS, Damas Argel de Melo C, Santos Noites AR, Machado JP, Gabriel MM. The effect of virtual reality on a home-based cardiac rehabilitation program on body composition, lipid profile and eating patterns: a randomized controlled trial. European Journal of Integrative Medicine. 2017;9:69-78

18. Vieira A, Gabriel J, Melo C, Machado J. Kinect system in home-based cardiovascular rehabilitation. Proc Inst Mech Eng H. 2017;231:40-7.

19. Daly J, Sindone A, Thompson D, Hancock K, Chang E, Davidson P. Barries to participation in and adherence to cardiac rehabilitation programs: A critical literature review. Prog Cardiovasc Nurs. 2002;17(1):8-17. 